\title{
Inhibitors of the Neutral Amino Acid Transporters ASCT1 and ASCT2 Are Effective in In Vivo Models of Schizophrenia and Visual Dysfunction
}

\author{
Yong-Xin Li, Jia-Ying Yang, Miguel Alcantara, Grigor Abelian, Ashutosh Kulkarni, \\ Ursula Staubli, and Alan C. Foster
}

Department of Biological Sciences, Allergan, Inc., Irvine, California

Received June 5, 2018; accepted August 27, 2018

\begin{abstract}
The N-methyl-D-aspartate receptor coagonist $\mathrm{D}$-serine is a substrate for the neutral amino acid transporters ASCT1 and ASCT2, which may regulate its extracellular levels in the central nervous system (CNS). We tested inhibitors of ASCT1 and ASCT2 for their effects in rodent models of schizophrenia and visual dysfunction, which had previously been shown to be responsive to D-serine. L-4-fluorophenylglycine (L-4FPG), L-4-hydroxyPG (L-4OHPG), and L-4-chloroPG (L-4CIPG) all showed high plasma bioavailability when administered systemically to rats and mice. L-4FPG showed good brain penetration with brain/plasma ratios of $0.7-1.4$; however,
\end{abstract}

values for L-4OHPG and L-4CIPG were lower. Systemically administered L-4FPG potently reduced amphetamine-induced hyperlocomotion in mice, whereas L-4OHPG was 100-fold less effective and L-4CIPG inactive at the doses tested. L-4FPG and L-4OHPG did not impair visual acuity in naive rats, and acute systemic administration of L-4FPG significantly improved the deficit in contrast sensitivity in blue light-treated rats caused by retinal degeneration. The ability of L-4FPG to penetrate the brain makes this compound a useful tool to further evaluate the function of ASCT1 and ASCT2 transporters in the CNS.

\section{Introduction}

Hypofunction of the $N$-methyl-D-aspartate (NMDA) subtype of glutamate receptors has been implicated in psychiatric and sensory disorders. The contribution of NMDA receptor hypofunction to schizophrenia was hypothesized initially in otherwise normal human subjects from the schizophrenia-like state induced by NMDA receptor antagonists, such as ketamine and phencyclidine (Javitt et al., 2012; Balu, 2016). Subsequently, the administration of the NMDA receptor coagonist D-serine was shown to relieve schizophrenic symptoms in patients when coadministered with antipsychotic agents (HerescoLevy et al., 2005; Kantrowitz et al., 2010), presumably by restoring NMDA receptor tone. In the visual system, NMDA receptors are important for responses along the visual pathway (Hartveit and Heggelund, 1990; Scharfman et al., 1990; Simon et al., 1992; Stevens et al., 2003), and we have shown previously that the administration of $\mathrm{D}$-serine can restore visual performance impaired by retinal degeneration (Staubli et al., 2016). Consequently, therapies based on restoring NMDA receptor tone may be effective in relieving schizophrenia symptoms and restoring visual function after retinal degeneration.

https://doi.org/10.1124/jpet.118.251116.
Although D-serine is a potent and effective coagonist of the NMDA receptor (Kleckner and Dingledine, 1988; Matsui et al., 1995), large doses are required to produce central effects after systemic administration because of poor blood-brain barrier permeability of this polar amino acid (Smith et al., 2009). In addition, high systemic levels of D-serine pose a risk of renal toxicity (Krug et al., 2007; Kantrowitz et al., 2010). We have previously shown that $\mathrm{D}$-serine is transported by the neutral amino acid transporters ASCT1 (SLC1A4) and ASCT2 (SLC1A5; Foster et al., 2016) and that the inhibition of these transporters can potentiate NMDA receptor-mediated neuronal plasticity in a D-serine-dependent manner (Foster et al., 2017). This suggests that these predominantly glial transporters may play an important role in regulating extracellular D-serine in the brain and thereby NMDA receptor function. Another approach has been to target the high-affinity transporters for the alternative endogenous NMDA receptor coagonist glycine. However, despite promising preclinical data, inhibitors of GlyT1 have not shown clinically meaningful benefits to date in schizophrenia patients (Singer et al., 2015; Bugarski-Kirola et al., 2016).

We previously identified phenylglycine (PG) analogs that are inhibitors of ASCT1 and ASCT2 (Foster et al., 2017), and here we tested their effects in animal models of schizophrenia and visual dysfunction to evaluate whether systemically

ABBREVIATIONS: ANOVA, analysis of variance; ASCT1, alanine/serine/cysteine transporter 1; ASCT2, alanine/serine/cysteine transporter 2; AUC, area under the curve; CL, clearance; $c$ d, cycles of grating per degree of visual angle; F, bioavailability compared with intravenous dosing; L-4CIPG, L-4-chlorophenylglycine; L-4FPG, L-4-fluorophenylglycine; L-4OHPG, L-4-hydroxyphenylglycine; LTP, long-term potentiation; NMDA, N-methyl-Daspartate; PG, phenylglycine; $T_{\max }$, time of $C_{\max } ; t_{1 / 2}$, half-life; VEP, visual evoked potential. 
administered inhibitors of these transporters had efficacy in these models of NMDA receptor hypofunction. The compounds evaluated were L-4-fluoroPG (L-4FPG) and L-4-chloroPG (L-4ClPG) which are relatively potent and selective ASCT1/2 inhibitors, and L-4-hydroxyPG (L-4OHPG) which additionally inhibits asc-1 (SLC7A10) (Foster et al., 2017), and has previously been tested in humans (Bergman et al., 1980). We previously showed that L-4FPG, L-4OHPG and L-4ClPG have $\mathrm{IC}_{50}$ 's for inhibition of astrocyte-mediated ASCT1 and ASCT2 transport of 44, 283 and $25 \mu \mathrm{M}$, respectively (Foster et al., 2017). They were not active in a range of ionotropic and metabotropic glutamate receptor assays and L-4ClPG and L-4FPG were tested and found to be inactive in a broader selectivity screen including 137 receptor, enzyme and transporter recognition sites (Foster et al., 2017).

\section{Materials and Methods}

\section{Animals}

All experiments were approved by the Allergan, Inc. (Irvine, CA) Institutional Animal Care and Use Committee and carried out in accordance with the Guide for the Care and Use of Laboratory Animals as adopted and promulgated by the U.S. National Institutes of Health.

\section{Materials}

L-4FPG and L-4OHPG were from Sigma-Aldrich (St. Louis, MO), and L-4ClPG was from Ark Pharm, Inc. (Arlington Heights, IL). All other laboratory agents were purchased from Sigma-Aldrich.

\section{Pharmacokinetic Studies}

Pharmacokinetic studies in rat for L-4FPG and L-4OHPG were performed by Allergan (Irvine, CA). Shanghai Medicilon Inc. (Shanghai, People's Republic of China) performed studies in mice for L-4FPG, L-4OHPG, and L-4ClPG.

Subcutaneous Administration of L-4FPG, L-4OHPG, and L-4ClPG to Mice. All compounds for subcutaneous administration were prepared for subcutaneous injection by dissolving them in water to yield nominal concentrations of 3 and $0.3 \mathrm{mg} / \mathrm{ml}$ (free form, $\mathrm{pH}$ 7) for L-4OHPG, 0.3 and $0.03 \mathrm{mg} / \mathrm{ml}$ for L-4FPG (free form, $\mathrm{pH}$ 7), and 60 and $30 \mathrm{mg} / \mathrm{ml}$ (free form, $\mathrm{pH}$ 7) for L-4ClPG. A total of 108 male C75BL/6 mice from Shangai Laboratory Animal Center (Shanghai Institutes for Biological Sciences, Shanghai, People's Republic of China) were subcutaneously administered a bolus dose of $10 \mathrm{ml} / \mathrm{kg}$, with varying concentrations ranging from 0.03 to $60 \mathrm{mg} /$ $\mathrm{ml}$ based on the compound administered. Plasma and brain tissue were subsequently collected and analyzed. Blood samples were collected at 15 minutes, 30 minutes, 1 hour, 2 hours, 4 hours, and 6 hours postdose. After centrifugation, the resulting plasma samples were transferred to clean tubes and stored frozen at $-80^{\circ} \mathrm{C}$ pending bioanalysis. After the blood collection, the brain of each animal was collected at 0.5 and 6 hours postdose. Prior to brain collection, perfusion with ice-cold normal saline was performed from the left ventricle. After perfusion, the brains were harvested, rinsed with saline, dried with filter paper, placed per animal into a tube, and frozen in dry ice then stored at $-80^{\circ} \mathrm{C}$ until bioanalysis. The concentrations of both compounds in matrix were determined using a high-performance liquid chromatography tandem mass spectrometry method. Individual and mean plasma and brain concentrations were reported, and WinNonlin Professional version 5.2 was used to calculate pharmacokinetic parameters area under the curve from time zero to last observation $\left(\mathrm{AUC}_{0-\mathrm{t}}\right)$, area under the curve from time zero to infinity $\left(\mathrm{AUC}_{0-\infty}\right)$, mean residence time from time zero to infinity $\left(\mathrm{MRT}_{0-\infty}\right),\left(t_{1 / 2}, \mathrm{~T}_{\max }, C_{\max }\right)$.
Administration of L-4FPG and L-4OHPG to Rat. Both compounds were prepared for subcutaneous, intraperitoneal, and intravenous injection by dissolving them in deionized water to yield a nominal concentration of $2.5 \mathrm{mg} / \mathrm{ml}$. A total of 18 precannulated fasted male Sprague-Dawley rats per compound were dosed with a bolus injection of $10 \mathrm{mg} / \mathrm{kg}$, i.v., i.p., or s.c., for L-4FPG; $10 \mathrm{mg} / \mathrm{kg}$, i.v.; or $30 \mathrm{mg} / \mathrm{kg}$, s.c. or i.p., for L-4OHPG. Plasma and brain tissues were subsequently collected and analyzed. Blood samples were collected at 5 minutes, 15 minutes, 30 minutes, 1 hour, 2 hours, 4 hours, 6 hours, and 8 hours after intravenous dosing. Blood samples were collected at 15 minutes, 30 minutes, 1 hour, 2 hours, 4 hours, 6 hours, and 8 hours after subcutaneous and intraperitoneal dosing. Approximately $0.3 \mathrm{ml}$ of blood at each time point was collected via the femoral cannula and placed into a tube with $\mathrm{K}_{3}$ EDTA. The blood was centrifuged to separate plasma and harvested into a 96 -well transfer plate. Brain samples were collected at 30 minutes postdose. Prior to brain collection, each rat was terminated via exsanguination under anesthesia. Brain was collected individually, and weight was recorded. The concentrations of both compounds in matrix were determined using high-performance liquid chromatography tandem mass spectrometry. Individual and mean plasma and brain concentrations were reported, and WinNonlin Professional version 5.2 was used to calculate pharmacokinetic parameters $\left[t_{1 / 2}, \mathrm{CL}\right.$, volume of distribution at steady state $\left(\mathrm{V}_{\mathrm{ss}}\right)$, and $\mathrm{AUC}_{0-\infty}$ values were calculated for the intravenous dose group; and $C_{\max }, \mathrm{T}_{\max }$, apparent half-life $\left(t_{1 / 2 \mathrm{app}}\right)$, and the percentage of bioavailability compared with intravenous dosing $(\%$ F) values were calculated for the subcutaneous and intraperitoneal dose groups].

\section{Amphetamine-Induced Hyperlocomotion}

C57B/6 male mice ( $n=5-10$ per group) were placed in an open field apparatus (PAS-Open Field; San Diego Instruments, San Diego, CA), and their activity was measured for 30 minutes. At 30 minutes, they received an injection with vehicle $(0.9 \% \mathrm{NaCl})$ or a test compound (subcutaneously) followed by an injection of amphetamine $(2 \mathrm{mg} / \mathrm{kg}$, s.c.) at minute 50 . Their activity levels were subsequently measured for another 2 hours after amphetamine injection. Total activity over the period from 50 to 120 minutes was used to establish an activity curve. Pre-amphetamine administration activity was measured over the period from 30 to 50 minutes. When testing D-serine or the PG analogs, tests for vehicle-injected animals were always run concurrently.

\section{Rats Sweep VEP Recording}

The methods were described in a previous article (Staubli et al., 2016). The recording electrodes were permanently implanted into the right visual cortex of Long-Evans rats at lambda and $4.5 \mathrm{~mm}$ lateral to the midline, to a depth of $800 \mu \mathrm{m}$ (layer III/IV). A reference electrode was placed epidurally on the midline $1.2 \mathrm{~mm}$ anterior to bregma. All recordings were conducted in awake rats starting at least 2 weeks after recovery from surgery. PowerDiva software from Anthony Norcia (Smith-Kettlewell Eye Research Institute, San Francisco, CA) was used for data acquisition and analysis. Visual stimuli were presented on a cathode ray tube computer monitor and consisted of full-field horizontally oriented sine-wave gratings at $80 \%$ contrast, reversing at $6.25 \mathrm{~Hz}$. VEPs were elicited by horizontally oriented gratings. The display was positioned $24 \mathrm{~cm}$ in front of the rat and centered at the vertical meridian. Mean luminance was held constant at $20 \mathrm{~cd}$. To determine visual acuity thresholds, the sweep consisted of 15 spatial frequencies increasing from 0.03 to 1.8 cycles of grating per degree of visual angle (cpd) in 15 linear steps at $80 \%$ contrast. To determine contrast thresholds in blue light-treated rats, a trial consisted of a contrast sweep increasing from $2.5 \%$ to $70 \%$ in 15 logarithmic steps, with the spatial frequency kept constant at predetermined values, typically at $0.575 \mathrm{cpd}$. The sweeps were averaged until the signal-tonoise ratio was at least 3 or above. Contrast sensitivity was calculated as the inverse of the contrast threshold. 
A

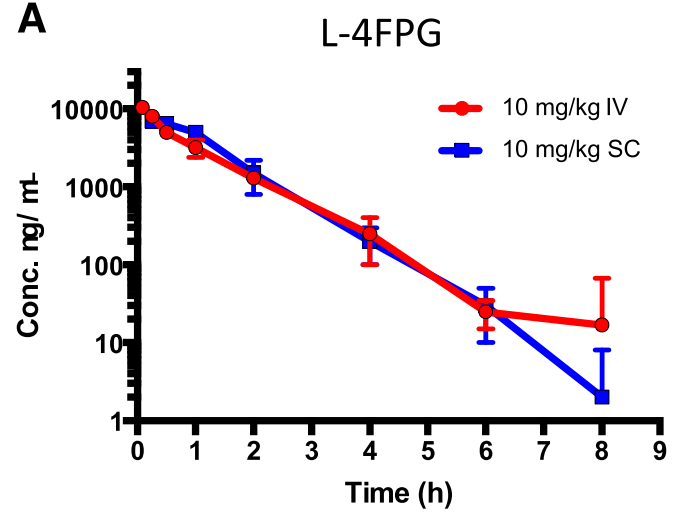

C

L-4FPG

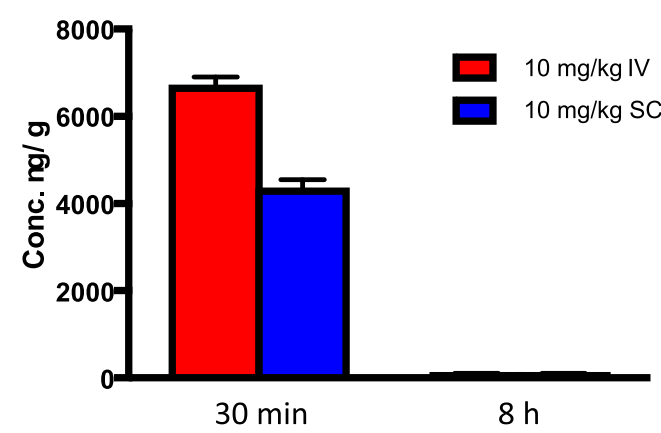

B

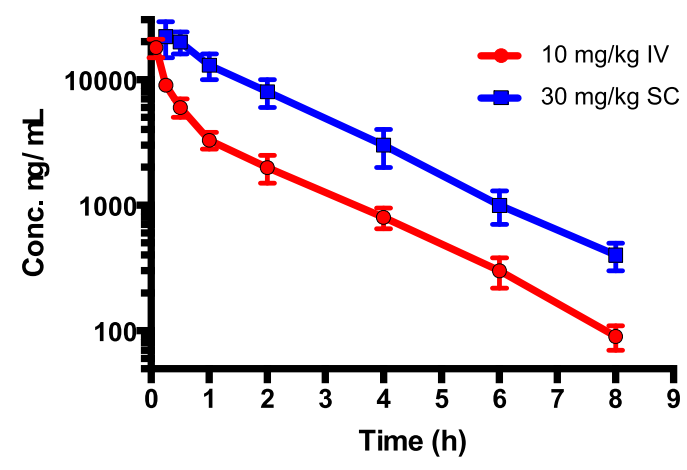

D

\section{L-4OHPG}

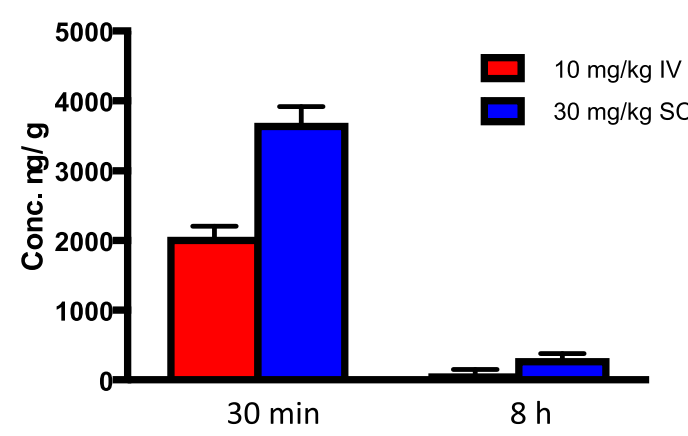

Fig. 1. Pharmacokinetics of L-4FPG and L-4OHPG in the rat after intravenous or subcutaneous administration. Plasma concentration-time profile of L-4FPG (A) and L-4OHPG (B) and brain concentration-time profile of L-4FPG (C) and L-4OHPG (D). PG analogs were administered at the indicated dose and route, and plasma, and brain samples were taken at various time points for the measurement of drug levels. Values are plasma or brain drug concentration and are the mean \pm S.D. of three animals per group. Conc., concentration.

Blue Light-Treated Rats. Young adult Long-Evans rats previously implanted with sweep VEP electrodes were used. They were dark adapted for 24 hours prior to exposing them to blue light consisting of $6000-8000 \mathrm{~lx}(\sim 460 \mathrm{~nm})$ for 8 hours. A subset of animals served as littermate controls and were returned to regular room light after dark adaptation. During the blue light exposure, all animals had access to gel food (blue light) or food and water ad libitum (room light). After blue light/ room light exposure, the animals were again dark adapted for 72 hours.

\section{Statistical Analysis}

Statistical analyses were performed using a one-way analysis of variance (ANOVA) with Dunnett's test for pairwise comparisons or with Student's $t$ test.

\section{Results}

Pharmacokinetic Studies with PG Analogs. The pharmacokinetics of $P G$ analogs were examined in mice and rats, the species used for evaluation of the effects of these compounds in models of schizophrenia and visual function. In addition to the assessment of bioavailability, a key question for these polar amino acid analogs was whether they could cross the blood-brain barrier sufficiently to influence nervous system function.

In rats, intravenous administration of L-4FPG indicated a relatively short plasma half-life $\left(t_{1 / 2}\right)(<1$ hour $)$, and rapid clearance $(\mathrm{CL})$; in comparison, the $t_{1 / 2}$ of L-4OHPG was longer, with slower CL and as a resulting plasma AUC that was greater than that of L-4FPG (Fig. 1; Table 1). Administration to rats by the subcutaneous route showed high plasma bioavailability with $\mathrm{F}$ values for both L-4FPG and L-4OHPG close to 1 (Fig. 1; Table 2). Administration of either compound by the intraperitoneal route also showed high bioavailability ( $F$ values $>0.8$ ) (Table 2). Measurement of brain levels 30 minutes after intravenous administration at $10 \mathrm{mg} / \mathrm{kg}$ indicated approximately $6 \mu \mathrm{g} / \mathrm{g}$ for L-4FPG (Fig. 1) with a brain-to-plasma ratio of 1.45; for L-4OHPG, lower brain levels (approximately $2 \mu \mathrm{g} / \mathrm{g}$ ) (Fig. 1) and brain-to-plasma ratio

TABLE 1

Plasma pharmacokinetics of PG analogs in the rat with intravenous administration

PG analogs were administered at the indicated dose, and plasma samples were taken at various time points for measurement of drug levels. Values are the mean \pm S.D. of three animals per group.

\begin{tabular}{lccccc}
\hline Compound & Dose, Route & $t_{1 / 2}$ & $\mathrm{CL}$ & $\mathrm{Vd}$ & AUC \\
\hline & $\mathrm{mg} / \mathrm{kg}$ & $\mathrm{h}$ & $\mathrm{ml} / \mathrm{min}$ per kilogram & $\mathrm{ml} / \mathrm{kg}$ & $\mathrm{ng} / \mathrm{h}$ per milliliter \\
L-4FPG & 10, i.v. & $0.82 \pm 0.12$ & $16.2 \pm 3.7$ & $889 \pm 48.0$ & $10,600 \pm 2380$ \\
L-4OHPG & 10, i.v. & $1.30 \pm 0.09$ & $11.6 \pm 1.1$ & $986 \pm 110$ & $14,500 \pm 1360$ \\
\hline
\end{tabular}

Vd, volume of distribution. 
TABLE 2

Plasma pharmacokinetics of PG analogs in the rat with subcutaneous and intraperitoneal administration

PG analogs were administered at the indicated dose and plasma samples taken at various time points for measurement of drug levels. Values are the mean \pm S.D. of three animals per group.

\begin{tabular}{lcccccc}
\hline Compound & Dose, Route & $t_{1 / 2}$ & \multicolumn{1}{c}{$C_{\max }$} & $\mathrm{T}_{\max }$ & $\mathrm{AUC}_{0-\infty}$ & $\mathrm{F}$ \\
\hline & $\mathrm{mg} / \mathrm{kg}$ & $\mathrm{h}$ & $\mathrm{ng} / \mathrm{ml}$ & $\mathrm{h}$ & $\mathrm{ng} / \mathrm{h}$ per milliliter \\
L-4FPG & 10, s.c. & $0.68 \pm 0.07$ & $7070 \pm 1070$ & $0.25 \pm 0.0$ & $10,200 \pm 1070$ & $0.97 \pm 0.24$ \\
L-4FPG & 10, i.p. & $1.04 \pm 0.78$ & $3940 \pm 3230$ & $1.58 \pm 2.10$ & $5800 \pm 3880$ & $0.93 \pm 0.62$ \\
L-4OHPG & 30, s.c. & $1.38 \pm 0.04$ & $21,900 \pm 4490$ & $0.33 \pm 0.14$ & $42,300 \pm 10,500$ & $0.96 \pm 0.10$ \\
L-4OHPG & 30, i.p. & $\mathrm{NC}$ & $27,400 \pm 2050$ & $1.24 \pm 0.05$ & $\mathrm{NC}$ & $0.83 \pm 0.08$ \\
\hline
\end{tabular}

NC, Not calculable because the terminal phase was not captured accurately.

(0.36) was observed (Table 3). At 8 hours postdose, both compounds showed low brain levels (Fig. 1). Brain-to-plasma ratios measured after subcutaneous or intraperitoneal administration in the rat also showed good brain penetration for L-4FPG, but lower values for L-4OHPG (Table 3).

In mice, subcutaneous administration of L-4FPG and L-4OHPG gave $t_{1 / 2}$ and $C_{\max }$ values for the two compounds that were consistent with the rat pharmacokinetics and resulted in a high plasma bioavailability (data not shown). Measurement of brain levels 30 minutes after subcutaneous administration in mice again indicated good brain exposure with a brain-to-plasma ratio of 0.73 for L-4FPG, and a lower value of 0.26 for L-4OHPG (Table 4). L-4ClPG was tested in mice by the intraperitoneal route and was found to have pharmacokinetic parameters similar to those for subcutaneously administered L-4FPG, including high plasma bioavailability (data not shown); however, the brain-to-plasma ratio for L-4CIPG was low (0.13) compared with that for L-4FPG (Table 4). These data indicate a high bioavailability by the intraperitoneal or subcutaneous route in rats and mice for the PG analogs and a more substantial brain penetration for L-4FPG compared with the other compounds. For L-4OHPG, brain penetration was more limited, but substantial brain exposure could be achieved by increasing the dose. By contrast, L-4ClPG had poor brain penetration. Consequently, the pharmacokinetic studies confirmed that single systemic doses of PG analogs were adequate for the evaluation of their effects in rodent models of schizophrenia and visual function where effects were monitored for up to 2 hours postdose.

Effects of PG Analogs in Amphetamine-Induced Hyperlocomotion. In clinical studies, D-serine has been shown to be effective in relieving the symptoms of schizophrenia (Kantrowitz et al., 2010). The PG analogs were tested in a mouse model of schizophrenia, where amphetamine induces hyperlocomotion. First, D-serine was tested by subcutaneous administration. Mice were allowed to habituate in the activity chamber for 30 minutes, at which time D-serine was injected subcutaneously followed 20 minutes later by injection of amphetamine at $2 \mathrm{mg} / \mathrm{kg}$, s.c., and activity was monitored for an additional 120 minutes. As shown in Fig. 2, hyperlocomotion induced by amphetamine was reduced in a dosedependent manner by D-serine (overall treatment effect was significant by one-way ANOVA, $F(4,51)=2.944 ; P=0.029)$; a post hoc Dunnett's test indicated that this effect was significant at the highest dose $(P=0.007)$. The high doses of D-serine required may reflect its low brain penetration and are similar to those reported previously in other in vivo rodent paradigms (Smith et al., 2009). To control for the possibility that D-serine may suppress locomotor activity in the absence of amphetamine, activity counts were summed during the 30- to 50-minute period after D-serine dosing and prior to amphetamine administration. As shown in Fig. 2, D-serine suppressed locomotor activity during this period (overall treatment effect was significant by one-way ANOVA, $F(4,51)=3.988 ; P=$ 0.007), and a post hoc Dunnett's test indicated that this effect was significant at the highest dose $(P=0.001)$. This suggests that some, if not all, of the effect of $\mathrm{D}$-serine on amphetamineinduced locomotion may be a nonspecific effect of D-serine to suppress locomotor activity. This highlights the difficulty in interpreting data with a compound like D-serine that has poor brain bioavailability, requiring high systemic exposure that may result in confounding nonspecific effects.

Using the same treatment paradigm, L-4FPG and L-4OHPG were tested for their effects on amphetamine-induced hyperlocomotion. For L-4FPG (Fig. 3), a dose-dependent reduction was observed (overall treatment effect was significant by oneway ANOVA, $F(4,34)=3.07 ; P=0.03)$ with a lowest effective dose for L-4FPG of $0.3 \mathrm{mg} / \mathrm{kg}$ (post hoc Dunnett's test, $P=$ 0.023 ); at $3 \mathrm{mg} / \mathrm{kg}$ the effect was not significant, indicating that a U-shaped dose-response relationship exists. Unlike D-serine, there was no effect of L-4FPG on the pre-amphetamine locomotor activity (the overall treatment effect was found to be nonsignificant by one-way ANOVA: $F(4,34)=0.753 ; P=$ 0.563) (Fig. 3). Similarly, L-4OHPG (Fig. 4) showed a dosedependent reduction of amphetamine-induced locomotor activity (the overall treatment effect was found to be significant by one-way ANOVA: $F(2,35)=3.30 ; P=0.049)$ with a lowest effective dose for L-4OHPG of $30 \mathrm{mg} / \mathrm{kg}$ (post hoc Dunnett's test, $P=0.032$ ). There was no effect of $L-4 O H P G$ on the pre-amphetamine locomotor activity (the overall treatment effect was found to be nonsignificant by one-way ANOVA:

\section{TABLE 3}

Brain penetration of $\mathrm{PG}$ analogs in rat after various routes of dosing PG analogs were administered at the indicated dose and route, and plasma and brain samples were taken at 30 minutes for the measurement of drug levels. The brain-toplasma ratio was calculated from the mean drug levels at 30 minutes. Data are from three animals per group.

\begin{tabular}{lcc}
\hline Compound & Dose, Route & Brain/Plasma Ratio \\
\hline & $\mathrm{mg} / \mathrm{kg}$ & \\
L-4FPG & 10, i.v. & 1.45 \\
L-4FPG & 10, s.c. & 0.66 \\
L-4FPG & 10, i.p. & 1.04 \\
L-4OHPG & 10, i.v. & 0.36 \\
L-4OHPG & 30, s.c. & 0.18 \\
L-4OHPG & 30, i.p. & 0.06 \\
\hline
\end{tabular}


TABLE 4

Brain penetration of $\mathrm{PG}$ analogs in mouse after various routes of dosing PG analogs were administered at the indicated dose and route, and plasma and brain samples were taken at 30 minutes for the measurement of drug levels. Values for brain levels are mean \pm S.D. of three animals per group. The brain-to-plasma ratio was calculated from the mean drug levels at 30 minutes.

\begin{tabular}{lccc}
\hline Compound Mouse & Dose, Route & Brain & Brain/Plasma Ratio \\
\hline & $\mathrm{mg} / \mathrm{kg}$ & $\mathrm{ng} / \mathrm{ml}$ & \\
L-4FPG & 3, s.c. & $919 \pm 198$ & 0.73 \\
L-4FPG & 0.3, s.c. & $90 \pm 21$ & 0.86 \\
L-4OHPG & 30, s.c. & $4520 \pm 529$ & 0.26 \\
L-4ClPG & 10, i.p. & $653 \pm 260$ & 0.13 \\
\hline
\end{tabular}

$F(2,35)=0.790 ; P=0.462$ ) (Fig. 4). In the amphetamine model, L-4ClPG was tested at 0.3 and $1 \mathrm{mg} / \mathrm{kg}$, the dose range where L-4FPG was shown to be effective. As shown in Fig. 5, there was no significant effect (unpaired, two-tailed Student's $t$ test) of L-4ClPG at 0.3 or $1 \mathrm{mg} / \mathrm{kg}$ on amphetamine-induced or pre-amphetamine locomotor activity.

Effects of PG Analogs in Models of Visual Function. Since we have previously shown that systemically applied D-serine can have beneficial effects on visual function (Staubli et al., 2016), L-4FPG and L-4OHPG were tested in naive rats for their ability to influence normal vision (Fig. 6). Compared with vehicle, L-4FPG (10 mg/kg, i.p.) produced a nonsignificant trend to improve visual acuity (two-tailed paired Student's $t$ test: vehicle, $P=0.973$; L-4FPG, $P=0.199$ ). $\mathrm{L}-4 \mathrm{OHPG}$ produced a small but significant improvement in visual acuity (two-tailed paired Student's $t$ test: vehicle, $P=0.566$; L-4OHPG, $P=0.011$ ). These data indicate that, at the doses tested, the PG analogs do not impair, but may enhance, visual acuity in naive rats.

We have previously reported that rats exposed to blue light have long-lasting and stable deficits in contrast sensitivity that can be acutely improved by systemic administration of D-serine (Staubli et al., 2016). In the current experiments, a group of rats was used 53 weeks after blue light exposure that had a stable contrast sensitivity deficit of approximately 6 (Fig. 6C); values for rats with intact vision under these experimental conditions were approximately 10 (Staubli et al., 2016). Administration of a single dose of L-4FPG $(10 \mathrm{mg} / \mathrm{kg}$, i.p.) 53 weeks after blue light exposure, when a stable visual deficit was present, produced an acute improvement in contrast sensitivity measured 30 minutes postdose (twotailed paired Student's $t$ test: vehicle, $P=0.832$; L-4FPG,

A
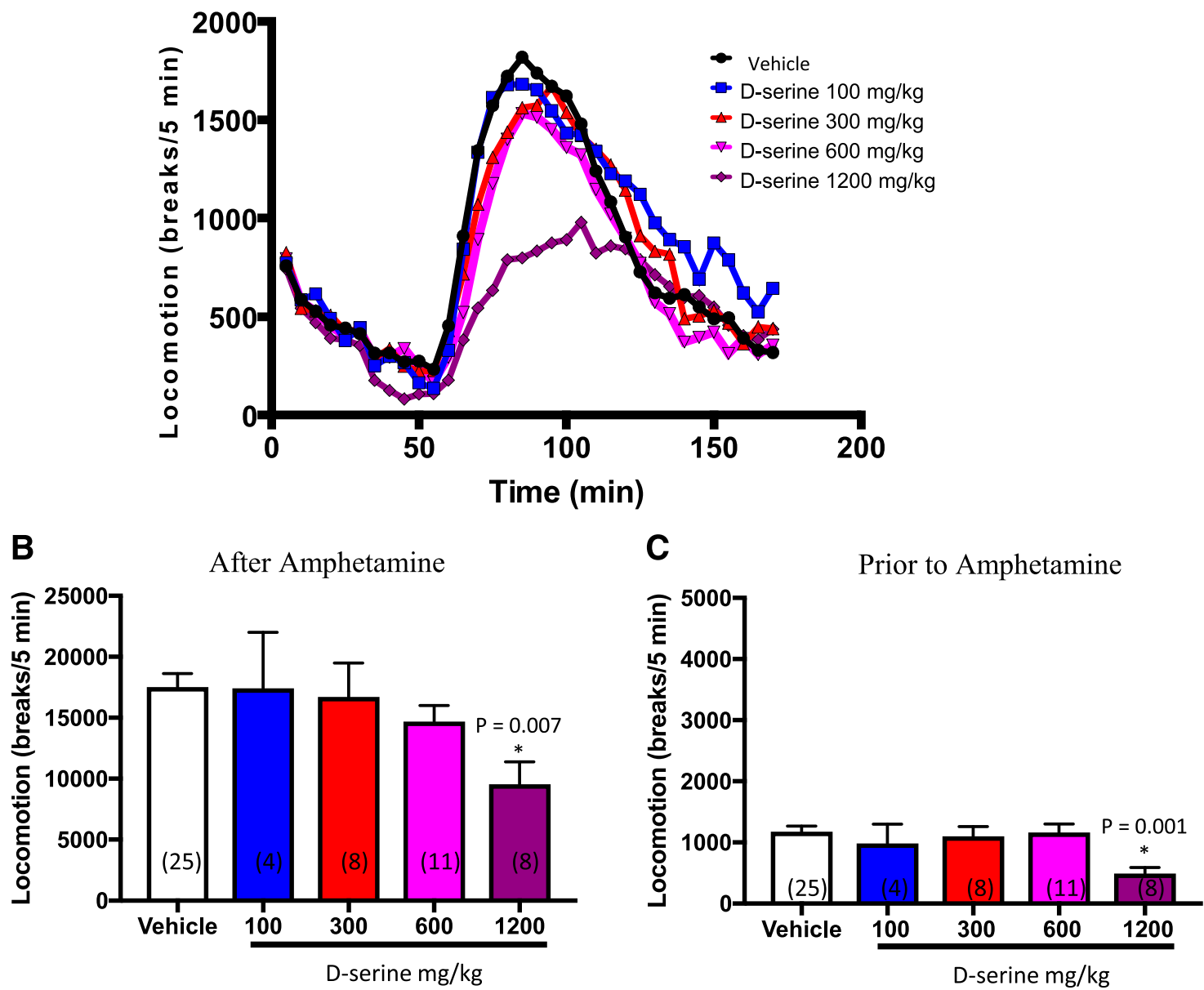

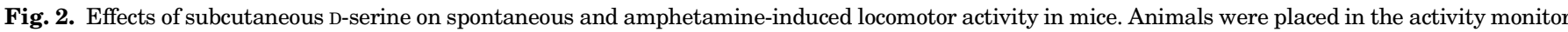

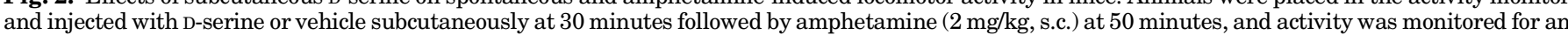

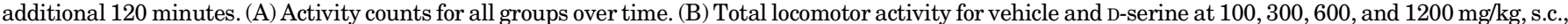

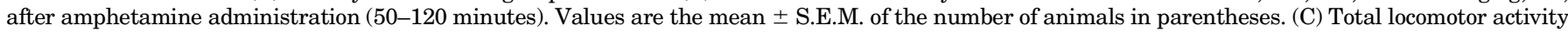

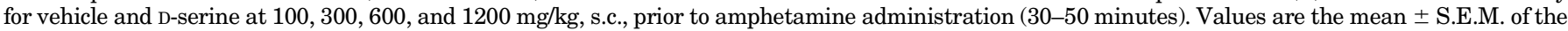
number of animals in parentheses. $P$ values are from post hoc Dunnett's test. *Significantly different from vehicle-treated animals. 

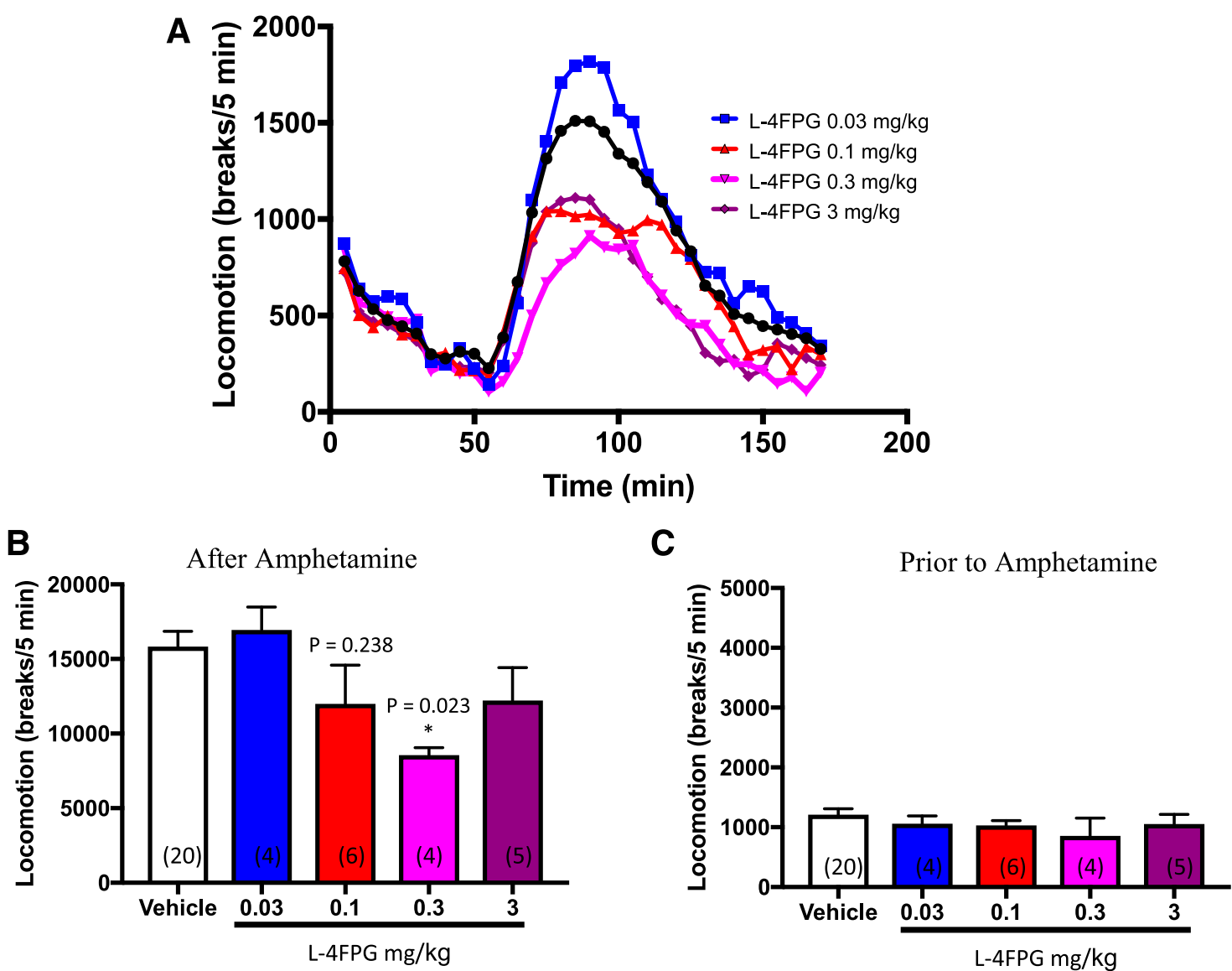

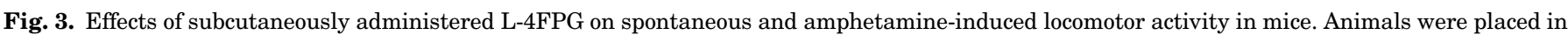

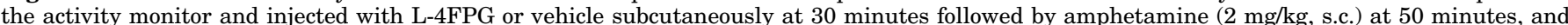

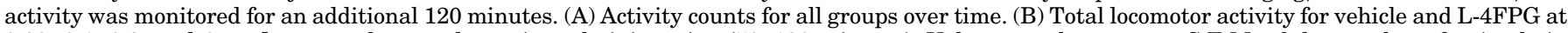

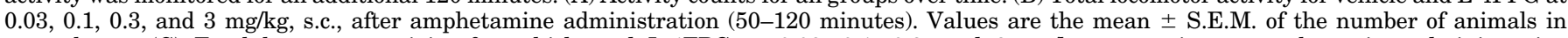

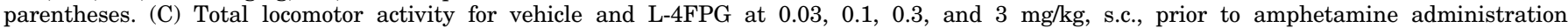

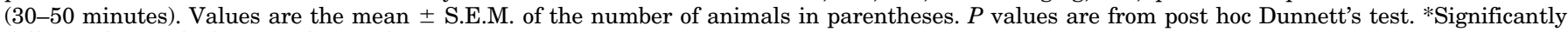
different from vehicle-treated animals.

$P=0.039$ ) (Fig. 6C). These data suggest that inhibition of ASCT1/2 in the visual system can acutely ameliorate the loss of visual function that results from retinal degeneration.

\section{Discussion}

The data presented here provide evidence that systemically administered inhibitors of ASCT1 and ASCT2 have effects in the brain and can ameliorate the NMDA receptor hypofunctional state that occurs in animal models of schizophrenia and visual dysfunction. Further optimization of PG analogs or other chemotypes reported to inhibit ASCT1 or ASCT2, such as benzylserines (Grewer and Grabsch, 2004), glutamine analogs (Esslinger et al., 2005; Schulte et al., 2015), and hydroxyprolines (Farnsworth et al., 2015) may lead to improved molecules with considerable therapeutic potential.

A concern at the outset of these studies was whether the PG analogs, which have an $\alpha$-amino acid structure and are polar in nature, would penetrate the blood-brain barrier to a degree that would allow them to be active in vivo after systemic administration. Pharmacokinetic studies in rats and mice indicated high bioavailability by intraperitoneal or subcutaneous routes, with $t_{1 / 2}$ values that allowed the evaluation of compound effects by a single acute administration in the animal models. Surprisingly, L-4FPG showed good bloodbrain barrier penetration, with brain-to-plasma ratios ranging from 0.7 to 1.4 in the rat and from 0.7 to 0.9 in the mouse. L-4OHPG and L-4ClPG had lower values. The 4-hydroxy moiety of L-4OHPG makes this the most polar of the three analogs; however, based on lipophilicity, L-4ClPG would be expected to have the best blood-brain barrier penetration [logP values calculated from the SwissADME website (http:// www.swissadme.ch/index.php) are L-4FPG $=0.09, \mathrm{~L}-4 \mathrm{ClPG}$ $=0.32, \mathrm{~L}-4 \mathrm{OHPG}=-0.65]$. Consequently, the favorable brain penetration of L-4FPG is likely due to factors other than lipophilicity. One intriguing possibility is that this molecule uses an amino acid transport system to cross the blood-brain barrier. A strong candidate is system L, a transporter for neutral amino acids present at the blood-brain barrier that is responsible for transporting several neuroactive amino acids, including L-DOPA and gabapentin (Kageyama et al., 2000; Dickens et al., 2013). PG analogs are substrates for system L (Reichel et al., 2000), and we previously showed that L-4FPG interacted with system L in astrocytes, whereas L-4OHPG and L-4ClPG were less active (Foster et al., 2017). The $\mathrm{IC}_{50}$ values of 36,233 , and $500 \mu \mathrm{M}$, respectively, for L-4FPG, L-4OHPG, and L-4CIPG for system L (Foster et al., 2017) correspond to the relative extent of brain penetration for these 

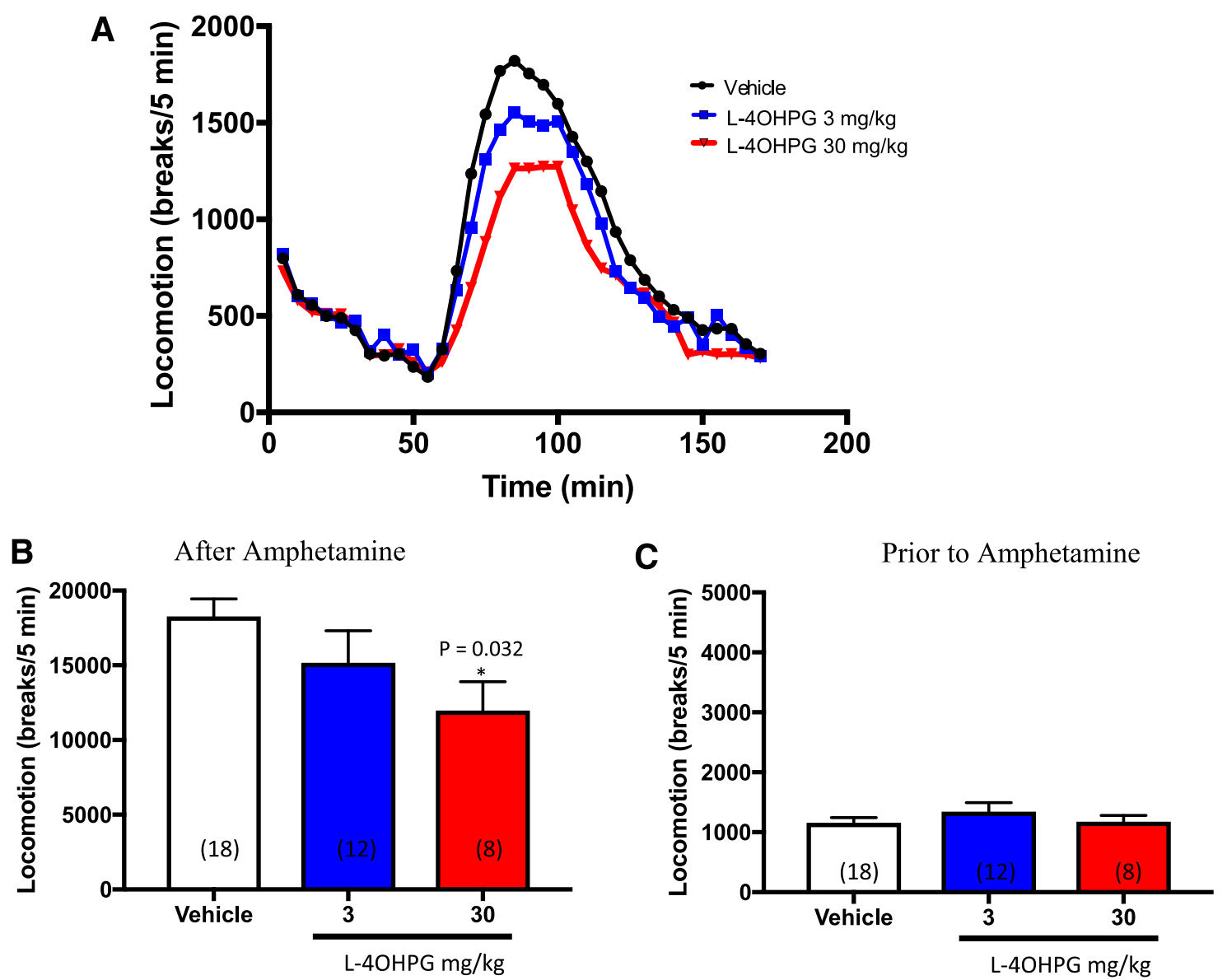

Fig. 4. Effects of subcutaneously administered L-4OHPG on spontaneous and amphetamine-induced locomotor activity in mice. Animals were placed in the activity monitor and injected with L-4OHPG or vehicle subcutaneously at 30 minutes followed by amphetamine (2 mg/kg, s.c.) at $50 \mathrm{minutes}$, and activity was monitored for an additional 120 minutes. (A) Activity counts for all groups over time. (B) Total locomotor activity for vehicle and L-4OHPG at 3 and $30 \mathrm{mg} / \mathrm{kg}$, s.c., after amphetamine administration (50-120 minutes). Values are the mean \pm S.E.M. of the number of animals in parentheses. (C) Total locomotor activity for vehicle and L- $4 \mathrm{OHPG}$ at 3 and $30 \mathrm{mg} / \mathrm{kg}$, s.c., prior to amphetamine administration (30-50 minutes). Values are the mean \pm S.E.M. of the number of animals in parentheses. $P$ values are from post hoc Dunnett's test. *Significantly different from vehicle-treated animals.

compounds, suggesting that system L may be an important factor in their blood-brain barrier permeability.

D-Serine has been shown to alleviate symptoms in schizophrenic patients (Kantrowitz et al., 2010) and has effects in preclinical animal models that may be predictive of clinical utility for schizophrenic symptoms (Contreras, 1990; Lipina et al., 2005; Smith et al., 2009). In addition, knockout of D-amino acid oxidase, the degradative enzyme for D-serine, results in elevated brain D-serine and an antipsychotic profile in mice (Hashimoto et al., 2008). In rats, Smith et al. (2009) showed that a high dose of D-serine $(1280 \mathrm{mg} /$ $\mathrm{kg}$ ) attenuated amphetamine-induced hyperlocomotion without effect on habituation in the absence of amphetamine. Our studies in mice showed a similar effect of high-dose Dserine $(1200 \mathrm{mg} / \mathrm{kg})$ on amphetamine-induced hyperlocomotion; however, this was accompanied by a reduction in spontaneous locomotor activity. This highlights a potential species difference in the effects of D-serine on spontaneous locomotor activity, but also raises the possibility that the effects of D-serine on amphetamine-induced locomotor activity were nonspecific and not related to central NMDA receptor activity. High systemic doses of D-serine are required to elevate central D-serine levels (Smith et al., 2009) because of the poor blood-brain barrier penetration of this polar amino acid. The good brain penetration of L-4FPG allowed this compound to be tested at relatively low doses in the mouse model. Indeed, L-4FPG showed a dose-dependent and potent effect on amphetamine-induced locomotor activity, with an optimal dose of $0.3 \mathrm{mg} / \mathrm{kg}$. This was not accompanied by any change in spontaneous locomotor activity. As the dose was increased to $3 \mathrm{mg} / \mathrm{kg}$, the effect was reduced; the reason for this is unclear. L-4OHPG also attenuated amphetamine-induced locomotor activity without an effect on spontaneous locomotor activity, but required a 100 times higher dose than L-4FPG. This may be due to reduced brain penetration combined with a weaker effect on ASCT1/2 transport (see below). At the same dose range where L-4FPG was effective, L-4ClPG $(0.3$ and $1 \mathrm{mg} / \mathrm{kg})$ had no effect on amphetamine-induced or spontaneous locomotor activity. Since L-4ClPG is equally effective with L-4FPG as an inhibitor of ASCT1/2, this suggests that the poor blood-brain barrier penetration of L-4ClPG (possibly reflecting that it is a poor substrate for system L; see above) did not allow it to show activity at the doses tested. Overall, the effects of the PG analogs in the mouse locomotor activity model support the idea that L-4FPG has central effects when applied systemically, which is consistent with its blood-brain barrier penetration and inhibition of ASCT1/2 transport. 
A
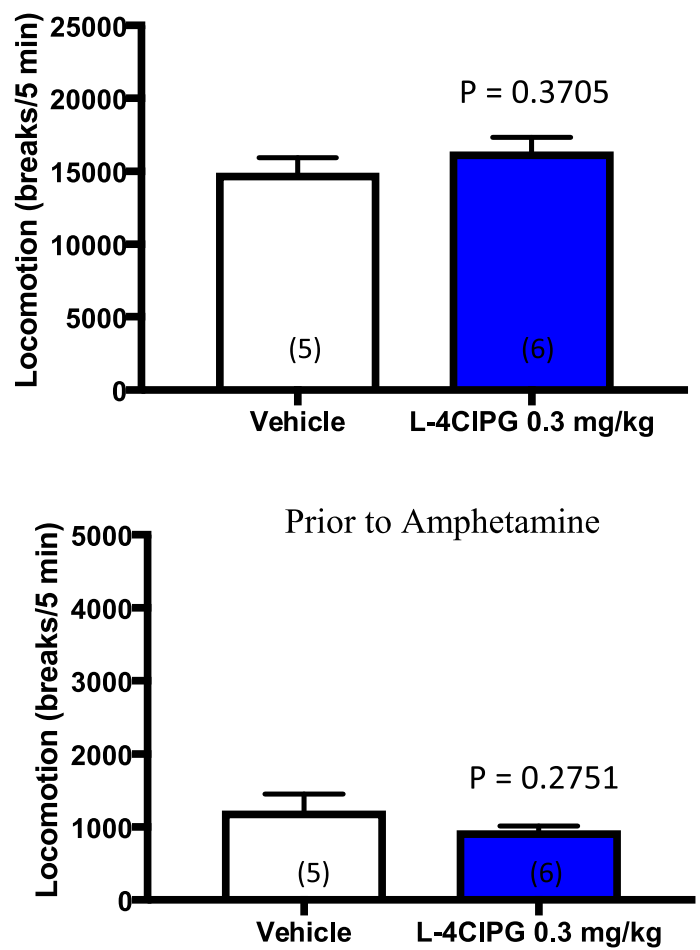

B

After Amphetamine
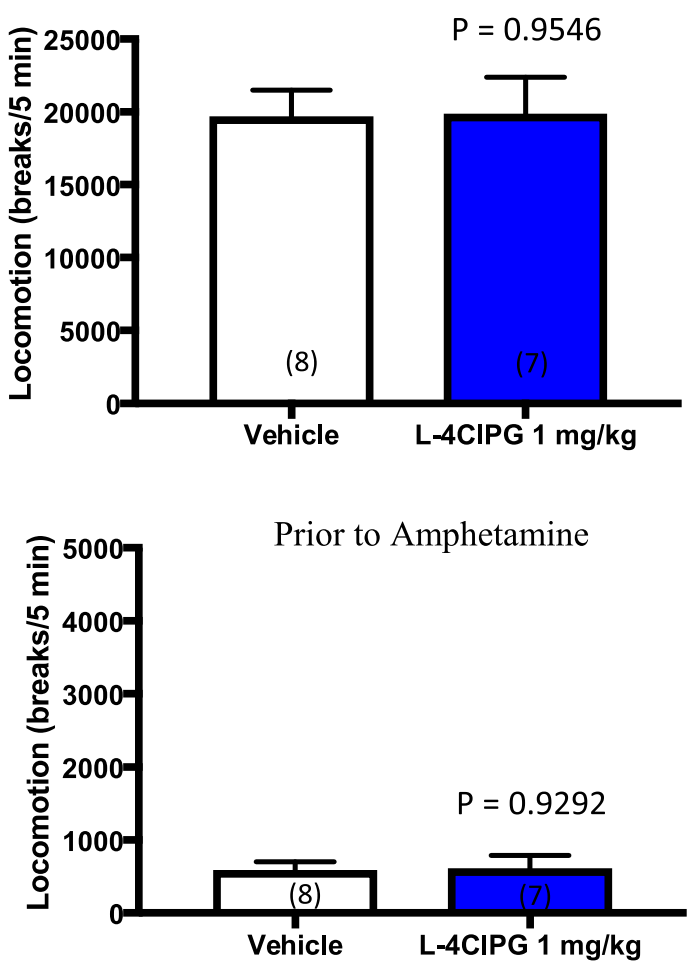

Fig. 5. Effects of SC L-4ClPG on spontaneous and amphetamine-induced locomotor activity in mice. Animals were placed in the activity monitor and injected with L-4ClPG or vehicle subcutaneously at 30 minutes followed by amphetamine $(2 \mathrm{mg} / \mathrm{kg}$, s.c.) at 50 minutes, and activity was monitored for an additional 120 minutes. Two independent experiments were conducted as shown in (A) and (B). (A, top panel) Total locomotor activity for vehicle and L-4ClPG at $0.3 \mathrm{mg} / \mathrm{kg}$, s.c., after amphetamine administration (50-120 minutes). (Bottom panel) Total locomotor activity for vehicle and L-4ClPG at $0.3 \mathrm{mg} / \mathrm{kg}$, s.c., prior to amphetamine administration (30-50 minutes). (B, top panel) Total locomotor activity for vehicle and L-4ClPG at $1 \mathrm{mg} / \mathrm{kg}$, s.c., after amphetamine administration (50-120 minutes). (Bottom panel) Total locomotor activity for vehicle and L-4ClPG at $1 \mathrm{mg} / \mathrm{kg}$, s.c., prior to amphetamine administration (30-50 minutes). Values are the mean \pm S.E.M. of the number of animals in parentheses. $P$ values are from unpaired twotailed $t$ tests.

The brain levels of L-4FPG and L-4OHPG in mice (measured at 30 minutes postdose) required to produce a significant reduction of amphetamine-induced locomotion were $90 \mathrm{ng} / \mathrm{ml}$ (at $0.3 \mathrm{mg} / \mathrm{kg} \mathrm{L}-4 \mathrm{FPG}$ ) and $4520 \mathrm{ng} / \mathrm{ml}$ (at $30 \mathrm{mg} / \mathrm{kg}$ L-4OHPG) (Table 4). These equate to brain concentrations of approximately 0.5 and $30 \mu \mathrm{M}$, respectively. We have previously shown that L-4FPG and L-4OHPG enhance LTP in rat visual cortex slices in a dose- and D-serine-dependent manner (Foster et al., 2017). In this paradigm, L-4FPG significantly increased LTP at $1 \mu \mathrm{M}$ with a trend at $0.3 \mu \mathrm{M}$, and L-4OHPG significantly elevated LTP at $100 \mu \mathrm{M}$ with a trend at $10 \mu \mathrm{M}$, reflecting their differing affinities for the ASCT1/2 transporters. Since the brain levels that were active in the amphetamine-induced locomotor activity assay are close to the concentrations of L-4FPG and L-4OHPG required to enhance NMDA receptor-dependent neuronal plasticity, it suggests that the inhibition of ASCT1/2 and subsequent enhancement of extracellular D-serine to enhance NMDA receptor activity is a plausible mechanism by which these compounds were effective in this in vivo model. For L-4ClPG, the lowest concentration producing a significant effect on LTP was $1 \mu \mathrm{M}$ (Foster et al., 2017). Extrapolating from the brain levels in mice achieved after intraperitoneal injection of $10 \mathrm{mg} / \mathrm{kg} \mathrm{L-4ClPG} \mathrm{(Table} \mathrm{4),} \mathrm{the} \mathrm{brain} \mathrm{levels} \mathrm{at} \mathrm{the} \mathrm{doses}$ tested in the mouse amphetamine model are approximately $0.35 \mu \mathrm{M}$ at $1 \mathrm{mg} / \mathrm{kg}$ and $0.11 \mu \mathrm{M}$ at $0.3 \mathrm{mg} / \mathrm{kg}$. Consequently, it appears that the brain levels achieved with the tested doses of L-4CIPG may be too low to inhibit ASCT1/2 sufficiently to produce an effect in vivo.

Our previous data from systemically administered D-serine provided evidence for an acute restoration of visual performance in animals with impaired vision resulting from retinal insults (Staubli et al., 2016). As an initial step in testing PG analogs, L-4FPG and L-4OHPG were tested with intraperitoneal administration for the effects on visual acuity in naive rats. Neither compound impaired vision, and L-4FPG showed a trend toward improvement, whereas, L-4OHPG significantly improved visual acuity (Fig. 6). Subsequently, L-4FPG was tested in rats with retinal impairment that have a long-term stable deficit in contrast sensitivity. A single dose of $10 \mathrm{mg} / \mathrm{kg}$, i.p., showed a significant improvement in contrast sensitivity measured 30 minutes postdose (Fig. 6). These data suggest that L-4FPG has the potential to improve visual function after retinal impairment at a dose that does not affect normal vision. This effect could be mediated through the enhancement of NMDA receptor function in central visual pathways or could be mediated at the level of the retina, where D-serine also has beneficial effects (Staubli et al., 2016).

In conclusion, we have provided evidence that PG analogs that are inhibitors of the neutral amino acid transporters ASCT1 and ASCT2 have central effects when administered systemically in animal models of schizophrenia and visual 

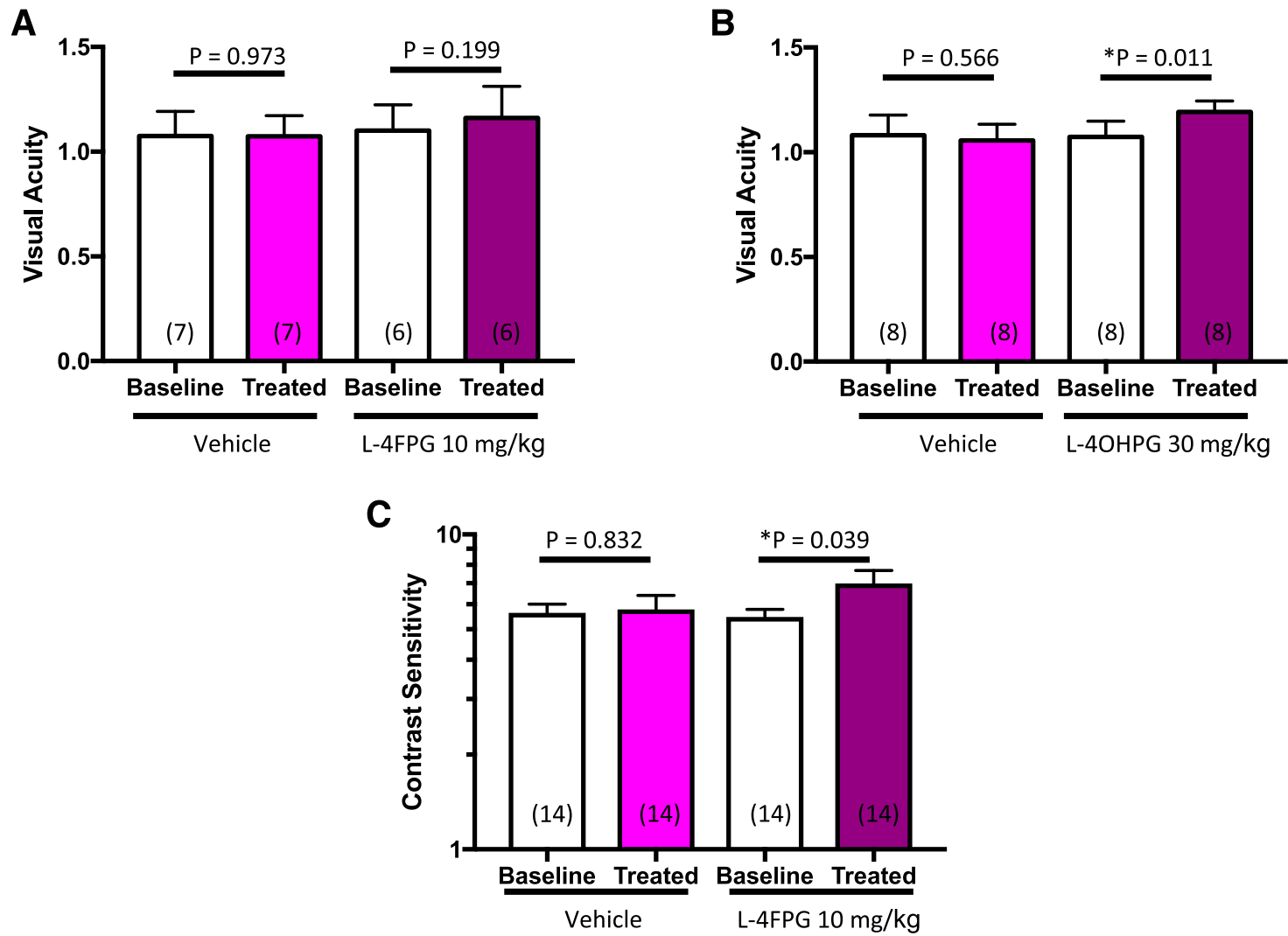

Fig. 6. Effects of L-4FPG and L-4OHPG on visual function in rats. Visual acuity (in cpd) was measured at baseline and then 30 minutes after the administration of vehicle or L-4FPG at $10 \mathrm{mg} / \mathrm{kg}$, i.p., (A) or vehicle or L-4OHPG at $30 \mathrm{mg} / \mathrm{kg}$, i.p., (B). (C) Rats were exposed to blue light to induce retinal degeneration and a stable deficit in contrast sensitivity (1/c, where $\mathrm{c}$ is contrast). Fifty-three weeks later, contrast sensitivity was measured at baseline and then 30 minutes after the administration of vehicle or L-4FPG at $10 \mathrm{mg} / \mathrm{kg}$, i.p. Values are the mean \pm S.E.M. of the number of animals in parentheses. $P$ values are from paired two-tailed $t$ tests. *Significantly different from baseline values.

dysfunction. In particular, L-4FPG has good brain penetration, which may be due to transport across the blood-brain barrier by system L. Compounds like L-4FPG or analogs with improved properties may have a therapeutic potential to treat disorders of NMDA receptor hypofunction.

\section{Authorship Contributions}

Participated in research design: Li, Kulkarni, Staubli, and Foster. Conducted experiments: Li, Yang, Alcantara, and Kulkarni.

Performed data analysis: Li, Yang, Alcantara, Abelian, Kulkarni, Staubli, and Foster.

Wrote or contributed to the writing of the manuscript: Li, Abelian, Kulkarni, Staubli, and Foster.

\section{References}

Balu DT (2016) The NMDA receptor and schizophrenia: from pathophysiology to treatment. Adv Pharmacol 76:351-382.

Bergman G, Atkinson L, Metcalfe J, Jackson N, and Jewitt DE (1980) Beneficial effect of enhanced myocardial carbohydrate utilisation after oxfenicine (Lhydroxyphenylglycine) in angina pectoris. Eur Heart $J$ 1:247-253.

Bugarski-Kirola D, Iwata N, Sameljak S, Reid C, Blaettler T, Millar L, Marques TR, Garibaldi G, and Kapur S (2016) Efficacy and safety of adjunctive bitopertin versus placebo in patients with suboptimally controlled symptoms of schizophrenia treated with antipsychotics: results from three phase 3, randomised, double-blind, parallel-group, placebo-controlled, multicentre studies in the SearchLyte clinical trial programme. Lancet Psychiatry 3:1115-1128.

Contreras PC (1990) D-serine antagonized phencyclidine- and MK-801-induced stereotyped behavior and ataxia. Neuropharmacology 29:291-293.

Dickens D, Webb SD, Antonyuk S, Giannoudis A, Owen A, Rädisch S, Hasnain SS and Pirmohamed M (2013) Transport of gabapentin by LAT1 (SLC7A5). Biochem Pharmacol 85:1672-1683.

Esslinger CS, Cybulski KA, and Rhoderick JF (2005) Ngamma-aryl glutamine analogues as probes of the ASCT2 neutral amino acid transporter binding site. Bioorg Med Chem 13:1111-1118.
Farnsworth JC, Lind GE, Lyda BR, Natale NR, and Kavanaugh MP (2015) SLC1A4 and SLC1A5 mediate transport of D-serine in brain, in Abstracts for Neuroscience 2015, the 45th Annual Meeting of the Society for Neuroscience; 2015 October 17-21; Chicago, IL. Vol. 41, pp 571.03, Society for Neuroscience, Washington, DC.

Foster AC, Farnsworth J, Lind GE, Li YX, Yang JY, Dang V, Penjwini M, Viswanath V, Staubli U, and Kavanaugh MP (2016) D-serine is a substrate for neutral amino acid transporters ASCT1/SLC1A4 and ASCT2/SLC1A5, and is transported by both subtypes in rat hippocampal astrocyte cultures. PLoS One 11:e0156551.

Foster AC, Rangel-Diaz N, Staubli U, Yang JY, Penjwini M, Viswanath V, and Li YX (2017) Phenylglycine analogs are inhibitors of the neutral amino acid transporters ASCT1 and ASCT2 and enhance NMDA receptor-mediated LTP in rat visual cortex slices. Neuropharmacology 126:70-83.

Grewer C and Grabsch E (2004) New inhibitors for the neutral amino acid transporter ASCT2 reveal its Na+-dependent anion leak. J Physiol 557:747-759.

Hartveit E and Heggelund P (1990) Neurotransmitter receptors mediating excitatory input to cells in the cat lateral geniculate nucleus. II. Nonlagged cells. J Neurophysiol 63:1361-1372.

Hashimoto A, Konno R, Yano H, Yoshikawa M, Tamaki R, Matsumoto H, and Kobayashi $\mathrm{H}$ (2008) Mice lacking d-amino acid oxidase activity exhibit marked reduction of methamphetamine-induced stereotypy. Eur $J$ Pharmacol 586: 221-225.

Heresco-Levy U, Javitt DC, Ebstein R, Vass A, Lichtenberg P, Bar G, Catinari S, and Ermilov M (2005) D-serine efficacy as add-on pharmacotherapy to risperidone and olanzapine for treatment-refractory schizophrenia. Biol Psychiatry 57: $577-585$

Javitt DC, Zukin SR, Heresco-Levy U, and Umbricht D (2012) Has an angel shown the way? Etiological and therapeutic implications of the PCP/NMDA model of schizophrenia. Schizophr Bull 38:958-966.

Kageyama T, Nakamura M, Matsuo A, Yamasaki Y, Takakura Y, Hashida M, Kanai Y, Naito M, Tsuruo T, Minato N, et al. (2000) The 4F2hc/LAT1 complex transports L-DOPA across the blood-brain barrier. Brain Res 879:115-121.

Kantrowitz JT, Malhotra AK, Cornblatt B, Silipo G, Balla A, Suckow RF, D'Souza C, Saksa J, Woods SW, and Javitt DC (2010) High dose D-serine in the treatment of schizophrenia. Schizophr Res 121:125-130.

Kleckner NW and Dingledine R (1988) Requirement for glycine in activation of NMDA-receptors expressed in Xenopus oocytes. Science 241:835-837.

Krug AW, Völker K, Dantzler WH, and Silbernagl S (2007) Why is D-serine nephrotoxic and alpha-aminoisobutyric acid protective? Am J Physiol Renal Physiol 293:F382-F390. 
Lipina T, Labrie V, Weiner I, and Roder J (2005) Modulators of the glycine site on NMDA receptors, D-serine and ALX 5407, display similar beneficial effects to clozapine in mouse models of schizophrenia. Psychopharmacology (Berl) 179 $54-67$.

Matsui T, Sekiguchi M, Hashimoto A, Tomita U, Nishikawa T, and Wada K (1995) Functional comparison of D-serine and glycine in rodents: the effect on cloned NMDA receptors and the extracellular concentration. $J$ Neurochem 65:454-458.

Reichel A, Begley DJ, and Abbott NJ (2000) Carrier-mediated delivery of metabotrophic glutamate receptor ligands to the central nervous system: structural tolerance and potential of the L-system amino acid transporter at the blood-brain barrier. J Cereb Blood Flow Metab 20:168-174.

Scharfman HE, Lu SM, Guido W, Adams PR, and Sherman SM (1990) N-methyl-Daspartate receptors contribute to excitatory postsynaptic potentials of cat lateral geniculate neurons recorded in thalamic slices. Proc Natl Acad Sci USA 87: $4548-4552$.

Schulte ML, Dawson ES, Saleh SA, Cuthbertson ML, and Manning HC (2015) 2Substituted $\mathrm{N} \gamma$-glutamylanilides as novel probes of ASCT2 with improved potency. Bioorg Med Chem Lett 25:113-116.

Simon DK, Prusky GT, O'Leary DD, and Constantine-Paton M (1992) N-methyl-D aspartate receptor antagonists disrupt the formation of a mammalian neural map. Proc Natl Acad Sci USA 89:10593-10597.
Singer P, Dubroqua S, and Yee BK (2015) Inhibition of glycine transporter 1: the yellow brick road to new schizophrenia therapy? Curr Pharm Des 21:3771-3787. Smith SM, Uslaner JM, Yao L, Mullins CM, Surles NO, Huszar SL, McNaughton CH Pascarella DM, Kandebo M, Hinchliffe RM, et al. (2009) The behavioral and neurochemical effects of a novel D-amino acid oxidase inhibitor compound 8 [4H-thieno [3,2-b]pyrrole-5-carboxylic acid] and D-serine. J Pharmacol Exp Ther 328:921-930.

Staubli U, Rangel-Diaz N, Alcantara M, Li YX, Yang JY, Zhang KM, and Foster AC (2016) Restoration of visual performance by d-serine in models of inner and outer retinal dysfunction assessed using sweep VEP measurements in the conscious rat and rabbit. Vision Res 127:35-48.

Stevens ER, Esguerra M, Kim PM, Newman EA, Snyder SH, Zahs KR, and Miller RF (2003) D-serine and serine racemase are present in the vertebrate retina and contribute to the physiological activation of NMDA receptors. Proc Natl Acad Sci USA 100:6789-6794.

Address correspondence to: Dr. Yong-Xin Li, Department of Biological Sciences, Allergan, Inc., 2525 Dupont Drive, Irvine, CA 92612. E-mail: li_yongxin@allergan.com; or Dr. Alan C. Foster, Otonomy Inc., 4796 Executive Drive, San Diego, CA 92121. E-mail: afoster@otonomy.com 